\title{
EQUITÀ E WELFARE STATE EUROPEO
}

\author{
LUIGI CAMPIGLIO (*)
}

SuNTO. - L'equità è un concetto fondamentale per l'efficiente funzionamento del meccanismo di mercato: vengono analizzati i limiti minimi e massimi dell'equità distributiva sul piano teorico per dimostrare come il funzionamento del mercato richiede il soddisfacimento di un limite minimo di dignitosa sussistenza, mentre il limite massimo è più difficile da individuare sul piano teorico. L'inconciliabilità fra equità ed efficienza è nella gran parte dei casi inesistente: maggiori salari aumentano infatti l'efficienza se il livello iniziale è molto basso, mentre salari crescenti lungo un percorso di carriera accrescono la motivazione dei lavoratori e la loro efficienza di gruppo. Il meccanismo di mercato può soddisfare le ragioni del merito, ma non rispondere, se non in modo strumentale attraverso il merito, alle ragioni del bisogno: il bisogno senza merito è tuttavia la caratteristica centrale di coloro la cui vita dipende da altri, come nel caso dei figli minori per i genitori o gli anziani non autosufficienti. Il Welfare State moderno è un'istituzione sociale in cui si rispecchiano i reciproci obblighi di equità, dalla famiglia nucleare alla famiglia nazionale, europea e umana. Nell'esperienza europea il Welfare è un'istituzione centrale in cui si rispecchiano i reciproci obblighi sociali e che ha dimostrato di funzionare in modo efficace nei momenti di crisi e a favore delle categorie più deboli.

ABSTRACT. - Equity is a fundamental concept for the efficient functioning of the market mechanism. The minimum and maximum limits of distributive equity are analysed on a theoretical level to demonstrate how the functioning of the market requires a minimum threshold of dignified subsistence, while the maximum limit is more difficult to locate in terms of theory. There is no incompatibility between equity and efficiency in most cases. Higher wages increase efficiency if the initial level is very low, while wage increases along a career path boost worker motivation and group efficiency. The market mechanism can satisfy the arguments relating to merit but does not respond to the arguments concerning need, except instrumentally in terms of merit. However, the need without merit is the central characteristic of those whose lives depend on others, as in the case of children in relation to their parents or the elderly who are not self-sufficient.

(*) Istituto Lombardo Accademia di Scienze e Lettere; Università Cattolica del sacro Cuore di Milano. Italia. E-mail: campiglio.universita@unicatt.it 
The modern Welfare state is a social institution that reflects the reciprocal obligations of equity, from individual family units, all the way to the national, European, and humanity 'family'. In the European experience, Welfare is a central institution that reflects reciprocal social obligations and has proven to function effectively in times of crisis, supporting the weakest categories.

\section{INTRODUZIONE}

L'equità è uno dei pochi valori che resistono alle trasformazioni culturali nel tempo, perché pur con differenti connotazioni rimane un riferimento centrale della vita politica, sociale ed economica: la forza collettiva che spinge a cambiare una situazione ingiusta è potente quanto quella che spinge a cercare la libertà. Ma l'equità, che in modo costitutivo nasce dal fatto della diversità, è lo strumento centrale per promuovere uno sviluppo stabile ed equilibrato. Per questo motivo è cruciale la ricerca di un consenso per intersezione non solo fra persone libere, ma anche fra differenti concezioni di equità, perché i confini di una concezione condivisa definiscono l'identità di una comunità umana, dalla sfera privata dei rapporti familiari a quella pubblica, di una nazione, dell'Europa come del genere umano. Nell'argomentazione che segue adottiamo la strategia di ricercare i confini per un comune ragionamento condivisibile, anziché fornire una definizione puntuale di equità sul piano economico.

\section{L’EQUITÀ: LIMITI MINIMI E MASSIMI DELLA DISUGUAGLIANZA}

Sul piano economico l'equità è di regola declinata come disuguaglianza e l'equità può essere definita come una disuguaglianza giusta, il che rimanda alla questione di quale sia la concezione di giustizia. Poiché abbiamo come obiettivo quello di individuare un'intersezione di consenso - anziché scegliere una specifica idea di giustizia - ci domandiamo se sia possibile individuare un limite minimo e massimo della diseguaglianza, essendo la disuguaglianza giusta collocabile fra questi limiti.

Consideriamo anzitutto il limite minimo, sul piano teorico, per quanto riguarda il funzionamento del mercato. Il mercato è un meccanismo decentrato di scambi per il quale è possibile dimostrare l'esistenza di un equilibrio efficiente, sulla base di alcune ipotesi di comporta- 
mento, oltre che di razionalità. Le relazioni fra i soggetti sono mediate da un solo segnale, quello dei prezzi, senza la necessità di alcuno scambio verbale, con la presenza di un soggetto esterno, una sorta di giudice di pace, il cui compito è di proporre un elenco di prezzi tale per cui la domanda di ciascun bene sia uguale - o non superiore - all'offerta per ogni soggetto. Affinché il "gioco dello scambio" si realizzi è quindi necessario che tutti i soggetti si presentino sul mercato con la capacità di domandare e offrire beni e servizi, cioè dispongano di una dotazione iniziale di beni, con un prezzo di mercato positivo, perché senza capacità di scambio non c'è equilibrio, efficienza e benessere sociale. Questa è una condizione minimale per il funzionamento efficiente del mercato. La dimostrazione teorica dell'efficienza del meccanismo di mercato dipende in modo essenziale dal fatto che ogni soggetto disponga di una dotazione iniziale di risorse, una condizione che sul piano teorico viene formulata come un'ipotesi preliminare variamente denominata, e che qui definiamo come "ipotesi di sopravvivenza". Il meccanismo dello scambio di mercato produce benefici aggiuntivi rispetto a quelli da cui ogni singolo soggetto potrebbe trarre vantaggio in modo "autarchico". L'ipotesi di sopravvivenza" pone tuttavia un problema centrale sul piano teorico e della politica economica, poiché con tale ipotesi si considera risolto un problema centrale che andrebbe invece dimostrato, e cioè la capacità del meccanismo di mercato di garantire la sopravvivenza a tutti i suoi partecipanti. La dotazione iniziale include la capacità di lavoro di ogni persona, e se esiste una domanda per tale capacità questa può essere considerata una dotazione iniziale: tuttavia il prezzo della dotazione di lavoro scende a zero se l'offerta di lavoro è maggiore della domanda. L'implicazione è quindi che in un mercato efficiente non vi è spazio per i working poor, cioè lavoratori che sono poveri perché il salario è così basso da non consentire loro una vita dignitosa.

In linea di principio si potrebbe dimostrare la possibilità di un equilibrio di mercato in cui i soggetti "scompaiono" come conseguenza di una dotazione di risorse iniziali insufficiente a garantire la sopravvivenza. Sul piano teorico il problema può essere risolto se esiste un soggetto che, come lo Stato, abbia il potere e la capacità di modificare l'allocazione delle risorse disponibili, ad esempio attraverso una manovra redistributiva di politica fiscale, vincolando il mercato alle allocazioni che consentano la sopravvivenza di ogni soggetto. Se, per semplicità, identifichiamo la sopravvivenza con il concetto di "povertà assoluta" ciò equivale ad affermare che lo Stato si deve porre come obiettivo 
quello di eliminare la "povertà assoluta", con appropriati strumenti di politica fiscale, come condizione necessaria per l'efficiente funzionamento del meccanismo di mercato.

La "guerra incondizionata contro la povertà", lanciata dal presidente Johnson nel suo messaggio al Congresso sullo Stato dell'Unione nel 1964, rappresenta una tappa importante nel processo di trasformazione moderna delle società, nella continuità dello slancio ideale del suo predecessore, John Kennedy, oltre che nel solco della tradizione di Roosevelt e della "libertà dal bisogno". Al centro della sua proposta Johnson pone l'idea delle "speranze di base": speranze di giuste opportunità, di un lavoro a tempo pieno per un salario pieno, di una casa per la famiglia in una decorosa comunità, di una buona scuola con buoni insegnanti, della sicurezza di un sostegno di fronte alla malattia, la disoccupazione o la vecchiaia. La grande sfida lanciata da Johnson ebbe un iniziale successo, con una diminuzione del tasso di povertà fino all'i $\mathrm{i}$ nizio degli anni '80, e poi un'ulteriore tendenziale diminuzione per le persone con oltre 65 anni, ma invece un ulteriore aumento per i giovani al di sotto dei 18 anni. L'amministrazione Clinton ha modificato nel 1996 la legislazione esistente nella direzione di offrire maggiori opportunità e incentivi di lavoro alle famiglie in condizioni di povertà, con il Personal Responsibility and Work Opportunity Act: nei dieci anni successivi la situazione della povertà non ha registrato sostanziali miglioramenti, ma la safety net, la rete di sicurezza sociale, nel frattempo costruita e migliorata a partire dagli anni ' 30 , ha consentito che la Grande Recessione del 2008 non si trasformasse nuovamente nella medesima tragedia umana del secolo scorso.

Mentre il limite minimo della disuguaglianza è individuabile nella soglia di una vita dignitosa, altrettanto non avviene per il limite massimo dell'utilità: sul piano teorico ciò corrisponde all'ipotesi di non sazietà, secondo la quale non esiste un "punto della felicità" perché il consumatore non è mai del tutto soddisfatto e appagato da ciò che già consuma o possiede. Ma il consumatore è anche un soggetto il cui reddito dipende dall'investimento in capitale umano, con un limite superiore governato dall'interazione fra domanda e offerta, e l'aumento della disuguaglianza può essere la conseguenza dell'incessante gara fra istruzione e tecnologia.

La recente ricostruzione storica della dinamica della disuguaglianza porta tuttavia in evidenza come il limite superiore sia governato da un processo più complesso e profondo. Per gli Stati Uniti, in cui esi- 
stono attendibili statistiche fiscali sui redditi fin dall'inizio del secolo scorso, è stato possibile ricostruire, da pochi anni, la quota di reddito percepito dall' $1 \%$ delle famiglie con il reddito più elevato: tale quota ha registrato onde lunghe di oscillazione nel corso dell'ultimo secolo, raggiungendo un massimo del $24 \%$ in due anni particolari, il 1928, cioè immediatamente prima della Grande Depressione, e il 2007, cioè immediatamente prima della Grande Recessione. In aggiunta si deve notare come nel periodo che va dal 1945 al 1975, segnato da rivolgimenti sociali ma anche da una lunga stagione di espansione economica e progresso sociale, la quota del top 1\% si stabilizzò intorno a un valore minimo del $10 \%$ circa. Il dibattito è aperto sul possibile nesso di causalità fra picco di disuguaglianza e crisi economico-strutturale, ma è indubbio che $\mathrm{i}$ due periodi che hanno preceduto la Grande Depressione e la Grande Crisi sono stati entrambi caratterizzati da un'esuberanza finanziaria sfuggita di mano e dall'accumulazione di ingenti fortune legate al mondo della finanza.

Nel caso dell'Italia, un paese con elevata disuguaglianza distributiva del reddito, le variazioni nel tempo hanno caratteristiche diverse: le famiglie consumatrici hanno registrato un'accentuata riduzione della quota di reddito sul Pil, a cui si è accompagnata una riduzione della capacità di risparmio; la capacità di reddito e di risparmio è influenzata in modo particolare dal fatto che nella coppia lavorino uno solo o entrambi, poiché un solo reddito medio non è più sufficiente a garantire una vita dignitosa e un'adeguata capacità di risparmio precauzionale. Per quanto riguarda la dinamica nel tempo della disuguaglianza, se consideriamo la variabilità dei livelli di consumo per decili - dato lo stretto legame fra consumi e reddito - possiamo osservare come la disuguaglianza dei consumi sia aumentata fra il 1997 e il 2007, e con l'inizio della crisi sia lievemente diminuita, seguendo una traiettoria analoga a quella americana.

Il meccanismo di mercato si muove perciò all'interno di limiti minimi e massimi, che se raggiunti richiedono entrambi un intervento dello Stato per consentire al mercato di riprendere a funzionare. Il meccanismo di mercato può consentire, in condizioni di effettiva concorrenza, di conseguire l'efficienza, mentre l'equità può essere realizzata solo come condizione strumentale per l'efficienza. L'assenza di mercati indispensabili per l'equità sociale, come i mercati assicurativi contro la disoccupazione, e l'instabilità associata alle eccessive disuguaglianze portano a concludere che vi sono obiettivi socialmente desiderabili - 
come la stabilità, l'assenza di eccessive disuguaglianze, l'irrilevanza dei bisogni nella formazione dei redditi primari, la mancanza di sicurezza di fronte alle incertezze di cambiamenti necessari - che il mercato non è strutturalmente in grado di ottenere: sono i market's SINS, un acronimo che vuole evocare tale inadeguatezza e la necessità di emendare il mercato dai suoi "peccati economici" con istituzioni come i sistemi di Welfare, nuovi o rinnovati.

\section{Merito, BISOGNO E OBBLIGAZIONI SOCIALI}

Il meccanismo di mercato può produrre efficienza, rispettando alcuni vincoli istituzionali, ma non può rispondere a bisogni che non siano sostenuti da un corrispondente potere di acquisto, come può accadere nel caso di persone povere, anziani oppure minorenni e bambini. Il mercato che funziona è quindi il terreno sociale che consente di ricompensare i meriti ma non i bisogni: quindi la domanda centrale che ci dobbiamo porre è come i meriti possano essere conciliati con i bisogni, o l'efficienza con l'equità. La famiglia è l'istituzione elementare nel cui ambito, e a certe condizioni, il merito si può accordare con il bisogno: il motivo ha radici nella teoria delle scelte sociali.

Come già ha argomentato Kenneth Arrow nel 1951, la famiglia rappresenta la forma più elementare di scelta collettiva, che funziona sulla base di "standard comuni di valore di qualche tipo". Come già aveva intuito Arrow, ed è poi stato dimostrato in anni recenti, il teorema di impossibilità delle scelte collettive è superabile quando le preferenze dei soggetti non siano troppo "distanti" fra di loro e ciò è proprio quanto ci si attende debba avvenire all'interno della vita di coppia che vada d'accordo. Una coppia stabile condivide di regola quel nucleo di valori comuni a cui Arrow faceva riferimento a proposito della famiglia: se accade che il marito preferisca la montagna e la moglie il mare, in una coppia che vada d'accordo non sarà difficile trovare un'intesa che soddisfi entrambi come famiglia, ad esempio alternando gite e vacanze al mare con quelle in montagna.

In una famiglia in cui uno o entrambi i genitori lavorano, le retribuzioni corrispondenti alle remunerazioni di mercato vanno a comporre un reddito familiare che viene redistribuito all' interno della famiglia sulla base dei bisogni di ciascun componente e non certo dei meriti di mercato. Un bambino non può avere alcun merito legato al mercato, 
mentre una moglie che si assume l'onere di curare la casa e i figli, e a volte anche i genitori non autosufficienti, ha una responsabilità e un impegno ancora più rilevanti di quello professionale, anche se non riconosciuto come merito dal mercato, perché non corrisponde a uno scambio monetario.

L'intuizione marxiana "da ciascuno secondo le sue capacità, a ciascun secondo i suoi bisogni” si realizza perciò all'interno delle famiglie che funzionano mentre - contrariamente alle attese di Marx - l'estensione di questo principio dalla famiglia allo Stato ha incontrato limiti insuperabili nell'esperienza storica. Lo Stato non è una famiglia. La risposta all'apparente contraddizione dipende dalla inadeguata considerazione di tutte le motivazioni umane ma - in modo più profondo è implicita nell'idea marxiana che il comunismo avrebbe rappresentato l'epoca della liberazione dai bisogni. Ma i bisogni considerati essenziali nel XXI secolo sono ben più ampi rispetto ai bisogni considerati essenziali alla fine dell' 800 , ed è a ciò che va ricondotto l'eccezionale aumento della speranza di vita nel corso del XX secolo: le infrastrutture dell'abitazione in cui viviamo, delle città in cui ci muoviamo, la qualità dei beni che consumiamo, delle medicine con cui ci curiamo - oggi rispetto a un secolo fa - tutto ciò ci fa comprendere, se appena ci riflettiamo, che i bisogni essenziali per vivere sono aumentati perché la durata e la qualità della nostra vita sono aumentate.

Se la sfera del merito è il dominio di un appropriato meccanismo di mercato, la sfera del bisogno è allora il dominio di un meccanismo di solidarietà sociale, come il Welfare State europeo o la Safety-net americana, sostenuti in modo essenziale da istituzioni quali la famiglia che, quando funziona, ricompone al proprio interno le ragioni del merito con quelle dell'efficienza. A livello macroeconomico questo processo corrisponde al problema di un possibile trade-off fra efficienza ed equità: l'argomento centrale è che una maggiore equità sociale potrebbe essere realizzata solo al prezzo di una minore efficienza economica. In concreto vi sono argomenti teorici ed evidenze empiriche che, a condizioni più qualificate, puntano invece in direzione opposta, cioè di un suo possibile superamento, come può avvenire nel caso della famiglia.

E' ormai acquisita l'idea che un aumento dei salari possa aumentare, anziché ridurre, la produttività, come accade in un paese agricolo arretrato in cui all'aumento dei salari corrispondono una salute migliore, maggiore forza fisica e quindi più produttività: l'equità si può quindi accompagnare all'efficienza, anzi esserne la causa. Ma ciò vale anche nel 
mondo delle imprese nei paesi avanzati, dove le indagini dimostrano con chiarezza come una corretta "giustizia procedurale" da parte del management favorisca la creazione di un clima di migliori rapporti fra i lavoratori, da cui dipende in modo decisivo il successo di qualunque impresa. La motivazione e la partecipazione dei lavoratori aumentano se esistono e vengono rispettate regole percepite come "eque", per gli aumenti di salario come per le progressioni di carriera.

Alla domanda se fosse meglio essere più temuto che amato Machiavelli propendeva per il timore, ma nel mondo moderno la paura diventa pura obbedienza, un freno alla libertà di pensare il nuovo, un limite invalicabile all'assunzione di responsabilità collettive: nelle imprese del XXI secolo è meglio essere rispettato che temuto, ma il rispetto si fonda sulla stima, la fiducia, la correttezza dei rapporti, in una parola sull'equità. La ragione pratica dell'equità è un tacito processo deliberativo con cui ciascuno riconosce i meriti degli altri rispetto a quelli propri, e i meriti premiati diventano così anche un modello esemplare di comportamento di ciò che la società riconosce come meritevole di ammirazione. L'ammirazione sociale, e non solo il denaro, è il motivo per cui il mondo della finanza ha attratto in questi anni una quota sempre più elevata di giovani talenti, anche se le vicende recenti hanno registrato una diminuzione dell'ammirazione sociale: a ciò, tuttavia, ancora non è seguito un parallelo adeguamento delle retribuzioni.

La crisi finanziaria ha portato in primo piano la dimensione centrale dell'equità in rapporto al rischio e all'incertezza: la questione centrale è la seguente. Le scommesse sul futuro sono inevitabili perché dal loro successo dipende lo sviluppo umano, ma come per tutti i giochi è fondamentale che la scommessa sia "equa": se a ciascuno dei 60 milioni di abitanti in Italia si richiede un contributo pro-capite per aumentare la probabilità di successo degli investimenti nella ricerca medica, tutti poi potranno accedere ai benefici delle nuove tecnologie, nel caso di successo. Analogamente lo scienziato che dovesse scoprire, con la fortuna guidata dall'ingegno, una nuova importante molecola, sarà (o dovrebbe essere) ricompensato e onorato in proporzione alle risorse che con la sua invenzione ha consentito di risparmiare. L'equità statistica è una guida centrale per consentire grandi scommesse sociali in nuova conoscenza.

Il nodo cruciale, sul piano dell'equità economica oltre che statisti$\mathrm{ca}$, è che i giocatori conoscano il gioco, siano consapevoli di giocare e, quando il gioco ha una qualche complessità, essi possano decidere 
avendo a disposizione tutte le informazioni consentite dalle regole del gioco. L'equità economica dipende, in questo caso, dall'equità informativa, cioè dal fatto che ogni parte sia a conoscenza di tutte le informazioni rilevanti per una decisione consapevole. Questa è proprio la condizione che è mancata negli anni che hanno preceduto la Grande Crisi, che dall'opacità informativa ha preso le mosse, poi rapidamente trasformatasi in una generalizzata dissonanza cognitiva, con il credere che uno strumento finanziario, spesso nuovo, rappresentasse un successo economico sicuro quando invece era fin dal concepimento predestinato al fallimento. La distribuzione delle informazioni fra le parti ha un ruolo decisivo nel loro successivo comportamento ed è anche la base per un "equo" indennizzo nei casi di scommesse senza successo.

L'invenzione sociale delle società di capitali, la cui responsabilità è limitata al patrimonio investito, ha prodotto benefici ma ha anche aperto un problema di responsabilità sociale per quanto riguarda gli errori "razionali" dell'imprenditore, cioè le decisioni dalle conseguenze sfortunate, il cui valore eccede il capitale investito e che comportano costi economici umani per i lavoratori coinvolti. Se il problema è generalizzato al sistema delle imprese, come nel corso della Grande Crisi, la crescita della disoccupazione e della precarietà del lavoro determina una perdita di valore economico del capitale umano che non sempre corrisponde all'indennità di fine rapporto. La distribuzione del rischio secondo criteri di equità, sociale e informativa, è uno strumento centrale per assorbire l'incertezza endogena dell'economia e attenuare l'impatto delle grandi crisi, vero banco di prova della stabilità del nostro sistema.

\section{IL WELFARE STATE: I NOSTRI RECIPROCI OBBLIGHI E I "CREDITORI PRIORITARI"}

Il Welfare State è un'istituzione sociale storicamente recente, che rispecchia l'evoluzione verso nuove forme istituzionali dei rapporti sociali, basate sul riconoscimento di appartenere a una medesima comunità, con reciproci obblighi di solidarietà ai quali ciascuno risponde con un proprio contributo, nella consapevolezza ed aspettativa che tutti gli altri si comportino allo stesso modo. Il Welfare State si fonda perciò sulla "conoscenza comune" di un medesimo comportamento che tutti si attendono da ciascuno e che ciascuno si attende da tutti: le 
ragioni dell'equità delimitano i mobili confini della comunità entro cui si decide di condividere un obbligo sociale.

Il fenomeno del free-rider non è un problema se la sua dimensione è limitata e soprattutto soggetta alla sanzione di una riprovazione sociale. Il fenomeno del free-rider può essere ulteriormente contenuto con forme di accertamento automatico, dalla fornitura "in natura" e soprattutto da un'informale "aria" informativa che, non diversamente da quanto accade per le imprese, produce inevitabilmente una "conoscenza comune". Il free-riding è, non casualmente, molto meno probabile all'interno di una famiglia, perché sono (o dovrebbero essere) reciprocamente conosciute le aspettative sulle obbligazioni reciproche e difficilmente un comportamento opportunistico può sfuggire allo sguardo della vita quotidiana. Le obbligazioni interne alla famiglia sono altresì un esempio evidente dell'esistenza di una gerarchia di obbligazioni, sia legali che affettive: in condizioni normali l'obbligazione di mercato ha una priorità sull'obbligazione interna, ma nei momenti di emergenza della vita familiare l'ordine di priorità si capovolge e le obbligazioni familiari vengono prima di quelle del mercato.

Se associamo ad ogni obbligazione un creditore ciò significa che non sempre è ragionevole che l'ordine di priorità dei creditori sia esclusivamente regolato dalle regole del mercato: è necessario un criterio di equità e ragionevolezza per individuare un equilibrio fra le ragioni del mercato espresse in contratti formali e le ragioni del bisogno e della solidarietà espresse dalle obbligazioni sociali, specialmente nel governo delle situazioni di emergenza. La nostra conoscenza del mondo, sia fisico che di relazioni umane, è spesso governata dalle crisi o dall'assenza, ed è così che ci rendiamo conto di possedere un muscolo quando ci duole, di aver bisogno di una persona quando ci manca, dell'importanza della pace quando c'è la guerra, ma anche del nostro bisogno di assistenza medica, e della sua importanza, quando ne abbiamo bisogno e invece ci manca.

Ciò che vale per un nucleo familiare vale anche, in modi diversi, per la famiglia umana, per la famiglia europea e per la famiglia di coloro con cui condividiamo da concittadini i medesimi valori nazionali. Esistono anche altri legami che ci legano a chi ci è vicino culturalmente, oltre che fisicamente, e la loro minor forza rispetto ai legami familiari è compensata dalla loro crescente densità, nello spazio e nel tempo, perché possiamo sentire obblighi reciproci che derivano dall'appartenere a una medesima nazione e dimostriamo di sentire obblighi anche nei con- 
fronti del passato, quando esprimiamo rispetto e ricordo per coloro che hanno contribuito a rendere migliore il mondo in cui viviamo.

Il Welfare State europeo è in realtà un mosaico di grandi aree di appartenenza culturale, quella dell'Europa del Nord, dell'Europa centrale, dell'Europa del Sud e della Gran Bretagna, ponte culturale fra Europa e Stati Uniti. La Grande Crisi ha scosso anche il modello americano di Welfare, la Safety-net, e l'importanza dei reciproci obblighi nei momenti di grave crisi non è mai stata così consapevole e presente come oggi: la spesa pubblica per la Safety-net americana è in costante crescita, come rapporto rispetto al Pil, da almeno trent' anni, dal 9\% nel 1980 al 15\% nel 2011 (considerando solo la social security e i programmi federali Medicare e Medicaid). Con la Grande Crisi la disoccupazione di lunga durata negli Stati Uniti ha raggiunto livelli mai registrati dalla fine della II Guerra Mondiale e, sia pur in modo ancora inadeguato, la rete di sicurezza è stata estesa per fare fronte all'emergenza.

In Europa la spesa per la protezione sociale - escludendo l'istruzione - rappresenta circa il 29\% del Pil dei paesi dell'area euro e quindi la spesa per il Welfare è la componente più strategica della politica fiscale, in particolare nel corso di severe crisi economiche, come è avvenuto nel 2009. Nel 2009 la spesa reale pro-capite per la protezione sociale nell'area euro è aumentata automaticamente del 6,6\%, mentre il Pil registrava una drastica diminuzione del $4,4 \%$, per poi aumentare in misura inferiore $(+0,7 \%)$ con la ripresa economica del $2010(+2 \%$ del Pil): a livello aggregato la spesa per il Welfare si è mossa perciò automaticamente in direzione anti-ciclica, assorbendo almeno parzialmente l'impatto economico dell'aumento della disoccupazione e della diminuzione del reddito familiare. L'effetto anti-ciclico non è stato tuttavia omogeneo fra i differenti paesi: in Germania la spesa reale pro-capite per la protezione sociale è aumentata del 7,9\% nel 2009, a fronte di una diminuzione del Pil del 5,1\%, mentre in Italia la spesa pro-capite per la protezione sociale nel 2009 è aumentata in misura inferiore della metà $(+3,4 \%)$, pur essendo la caduta del Pil ancora più elevata $(-5,5 \%)$. La ripresa economica della Germania nel 2010 è stata, anche per questo motivo, molto più elevata di quella dell'Italia.

L'efficacia della spesa per la protezione sociale può essere valutata misurando l'incidenza della povertà economica prima e dopo l'intervento redistributivo della spesa sociale (la povertà economica è misurata come il $60 \%$ del reddito mediano equivalente): le differenze di efficacia della spesa di protezione sociale fra i paesi europei sono tuttavia 
elevate. Nel 2010 la stima della povertà in Francia prima dei trasferimenti sociali era pari al $25 \%$ della popolazione e diminuiva al $13,5 \%$ dopo i trasferimenti, cioè con una diminuzione di 11,5 punti (misura della sua efficacia): in Italia il livello della povertà scendeva dal 23,3\% (prima dei trasferimenti) al 18,2\% (dopo i trasferimenti) con una più contenuta diminuzione di 5,1 punti. L'efficacia della spesa per la protezione sociale, misurata come riduzione della povertà economica, è quindi molto più elevata in Francia che in Italia, dove è più focalizzata sulla fascia sociale più nettamente svantaggiata, cioè bambini e ragazzi minorenni. Se ci poniamo la domanda di quali siano le categorie di trasferimenti che hanno la maggiore efficacia, misurata come riduzione della povertà fra prima e dopo i trasferimenti sociali, il confronto fra tutti i paesi europei dimostra come, non casualmente, l'efficacia sia molto più elevata nei paesi in cui è più elevata la quota di spesa sociale per la famiglia e i bambini, la sanità e la disabilità.

I sistemi di Welfare influiscono perciò in modo decisivo su una forma di equità che è al fondamento di tutte le moderne democrazie liberali, e cioè lo sforzo di garantire una condizione di uguaglianza di opportunità alla "partenza". La domanda cruciale è naturalmente in quale momento della vita si debba collocare il nastro della partenza. L'uguaglianza sarebbe solo nominale se il nastro fosse collocato alla maggiore età, cioè dopo i 18 anni, perché a quell'età le caratteristiche centrali di un giovane sono già formate. Il nastro deve invece essere collocato almeno al momento della nascita, ed è in base a questa consapevolezza, forse intuitiva, che negli Stati Uniti è stato creato un programma federale dedicato alle madri e ai bambini con basso reddito (Women, Infants, and Children - WIC), nel tempo rivelatosi come uno dei più efficaci.

Si tratta di un programma che tocca al cuore il funzionamento delle moderne democrazie, perché tutti gli studi psicologici documentano come i tratti centrali delle qualità fisiche, cognitive e comportamentali dei futuri cittadini si modellano nei primi cinque anni di vita, al più dieci. L'equità distributiva nei primi dieci anni di vita si traduce in risorse che hanno la caratteristiche di investimenti irreversibili e coinvolgono due fondamentali agenzie formative, i genitori e la scuola. I nostri reciproci obblighi nei confronti dei futuri cittadini dovrebbero avere perciò la forma di una priorità assoluta dei "crediti" dei giovani, se quando la classe politica dichiara di avere un debito nei loro confronti crede davvero in ciò che afferma. Il legittimo dubbio nasce dal fatto 
che trascurare i minorenni non influisce sui risultati elettorali, come già ricordava John Stuart Mill, e il politico lungimirante nei confronti dei futuri cittadini rischia - paradossalmente - di perdere il suo seggio in Parlamento. E quindi sarà costretto ad adeguarsi a tutti gli altri, diventandone indistinguibile.

La categoria dei creditori prioritari riguarda altresì le persone "sfortunate", che, pur avendo partecipato con correttezza alle scommesse sociali, ne sopportano senza alcuna colpa le eventuali conseguenze negative: così come avviene per chiunque riceva in eredità, senza sua colpa, un DNA difettoso. Una comunità ha obblighi naturali verso chi non può pienamente partecipare alla vita economica e sociale a causa di disabilità: anch'essi sono creditori prioritari.

La categoria dei creditori prioritari dipende dall'estensione della comunità di riferimento, il che comporta obblighi naturali nel caso della famiglia e obblighi sociali nei confronti dei concittadini di una medesima nazione: la natura e la forza delle reciproche obbligazioni all'interno dell'Europa rappresentano oggi la questione centrale per il futuro dell'Unione Europea, perché in una fase di crisi le priorità delle obbligazioni sociali fra i cittadini europei si intrecciano con le priorità dei crediti finanziari ed è quindi necessario individuare un criterio di scelta, o un compromesso accettabile, fra le due priorità. Se dovesse prevalere solo il credito finanziario, perché più forte, ciò significherebbe rinunciare al cemento sociale di reciproche obbligazioni fondate sulla solidarietà e la storia ci ricorda come il prevalere dei creditori forti è raramente la premessa di uno sviluppo equilibrato e sostenibile.

Un Welfare inteso come strumento di equità può invece consentire al meccanismo di mercato di realizzare in modo efficiente obiettivi di crescita e sviluppo sostenibile, stabilizzando i redditi delle famiglie e la loro capacità di risparmio, con cui finanziare gli investimenti e promuovere lo sviluppo del paese: la Francia, la Germania e la Svezia sono esempi di differenti sistemi di Welfare, che producono risultati positivi su molti piani, mentre nel caso dell'Italia e della Gran Bretagna vi è molto spazio per miglioramenti: le innovazioni sociali del XX secolo si sono diffuse con meccanismi di imitazione, pur essendo l'Europa divisa, quando non in conflitto armato, e la crisi economica attuale rappresenta un'opportunità per rafforzare la solidarietà europea, realizzando una convergenza dei legami sociali come premessa di quelli economici. 